\title{
A literature review on the representativeness of randomized controlled trial samples and implications for the external validity of trial results
}

Tessa Kennedy-Martin ${ }^{1 *}$, Sarah Curtis ${ }^{2}$, Douglas Faries ${ }^{2}$ Susan Robinson ${ }^{1}$ and Joseph Johnston ${ }^{2}$

\begin{abstract}
Randomized controlled trials (RCTs) are conducted under idealized and rigorously controlled conditions that may compromise their external validity. A literature review was conducted of published English language articles that reported the findings of studies assessing external validity by a comparison of the patient sample included in RCTs reporting on pharmaceutical interventions with patients from everyday clinical practice. The review focused on publications in the fields of cardiology, mental health, and oncology. A range of databases were interrogated (MEDLINE; EMBASE; Science Citation Index; Cochrane Methodology Register). Double-abstract review and data extraction were performed as per protocol specifications. Out of 5,456 de-duplicated abstracts, 52 studies met the inclusion criteria (cardiology, $n=20$; mental health, $n=17$; oncology, $n=15$ ). Studies either performed an analysis of the baseline characteristics (demographic, socioeconomic, and clinical parameters) of RCT-enrolled patients compared with a real-world population, or assessed the proportion of real-world patients who would have been eligible for RCT inclusion following the application of RCT inclusion/exclusion criteria. Many of the included studies concluded that RCT samples are highly selected and have a lower risk profile than real-world populations, with the frequent exclusion of elderly patients and patients with co-morbidities. Calculation of ineligibility rates in individual studies showed that a high proportion of the general disease population was often excluded from trials. The majority of studies ( $n=37$ [71.2\%]) explicitly concluded that RCT samples were not broadly representative of realworld patients and that this may limit the external validity of the RCT. Authors made a number of recommendations to improve external validity. Findings from this review indicate that there is a need to improve the external validity of RCTs such that physicians treating patients in real-world settings have the appropriate evidence on which to base their clinical decisions. This goal could be achieved by trial design modification to include a more representative patient sample and by supplementing RCT evidence with data generated from observational studies. In general, a thoughtful approach to clinical evidence generation is required in which the trade-offs between internal and external validity are considered in a holistic and balanced manner.
\end{abstract}

Keywords: Randomized controlled trial, External validity, Generalizability, Real-world patients, Cardiology, Mental health, Oncology, Literature review

\footnotetext{
* Correspondence: Tessa@kmho.co.uk

${ }^{1}$ Kennedy-Martin Health Outcomes Ltd, 3rd Floor, Queensberry House, 106

Queens Road, Brighton BN1 3XF, UK

Full list of author information is available at the end of the article
} 


\section{Background}

Appropriately designed and executed randomized controlled trials (RCTs) represent the current gold-standard primary study design for the determination of the efficacy and safety of medical interventions [1]. Evidence from RCTs is used by healthcare providers to guide their clinical decisions and by payers and policy makers to support their recommendations for the adoption of new therapies in clinical practice [2]. Explanatory RCTs are designed to determine the efficacy of an intervention under idealized and controlled circumstances and so are conducted under rigorous conditions, including strict adherence to structured protocols, the use of restrictive inclusion and exclusion criteria, and patient randomization, that maximize their internal validity (that is to ensure they minimize the possibility of bias regarding the effect of an intervention) $[3,4]$. In order for the results of such trials to be clinically useful, they must also be relevant to a definable patient population in a specific healthcare setting, a concept that is termed external validity or generalizability (note, these terms are used interchangeably [3] in this review and describe the applicability of the study results outside of the trial environment) [5-7]. As it is challenging to simultaneously optimize internal and external validity, efficacy data from traditional explanatory RCTs are often complemented by evidence from pragmatic trials (including pragmatic RCTs) or observational studies that determine the performance of an intervention under conditions more closely resembling routine clinical practice, and include more heterogeneous patient populations and less stringent treatment and delivery protocols [4]. While some pragmatic trials have good internal validity and some observational studies may lack external validity, generally explanatory RCTs tend to maximize internal validity at the expense of external validity, while studies conducted in a setting more closely resembling real-world practice may do the opposite. As such, evidence from all these sources can be complementary in understanding the effect of an intervention and furthering clinical research [8].

In recent years, the need to better understand the external validity of RCT results has been identified across numerous therapeutic areas [9-13]. However, a comprehensive literature review of studies that have assessed the representativeness of RCT populations has not been undertaken in recent years (note, the term representativeness has been used throughout this review to describe the similarities between RCT samples and realworld populations). To examine this issue, we conducted a literature review of studies that have attempted to evaluate external validity in one of two ways: (i) by comparing the clinical characteristics of an RCT sample with those of everyday clinical practice patients, or (ii) by assessing what proportion of a real-world population would satisfy the criteria for RCT inclusion. In the context of the current review, real-world populations are defined as those patients encountered in routine clinical practice settings (for example, patients included in observational cohorts or patients identified from medical chart review, registries, or insurance databases). The primary objective of the review was to assess the extent to which RCT samples are representative of real-world populations (which may or may not affect the external validity of the trial findings). Other objectives were to identify key issues that may impact the external validity of trial findings (with reference to included studies) and also to outline recommendations from the identified studies for improving external validity. The present review was limited to RCTs in oncology, mental health, and cardiology as, when the review was undertaken, these were identified as the main therapeutic areas in which RCT and real-world populations had been compared. It should be noted that the focus of the current review was explanatory and not pragmatic RCTs.

\section{Review \\ Methods}

The methodological framework of this literature review was employed to examine the extent, range, and nature of research activity regarding the representativeness of RCT patient samples and the implications of this to the external validity of the findings. The review involved a five-stage process [14]: identification of the research question; identification of studies relevant to the research question; selection of studies to include in the review; charting of information and data within the included studies; collating, summarizing, and reporting results of the review. A search protocol was written that outlined the objectives, search methods, and the process for study selection and data extraction.

\section{Information sources, search approach, and strategy}

Searches were run in MEDLINE and MEDLINE InProcess, EMBASE, Science Citation Index, and the Cochrane Methodology Register and were supplemented with reference checking. When combined with citation searching, these sources presented a reasonable basis for a targeted search of the published literature. The searches were run on 30 September 2013 and included published studies conducted from 2003 to 2013 in order to reflect contemporary clinical trial practice. A basecase search strategy was created in the Ovid MEDLINE interface, and once finalized, was adapted to meet the syntax of the other databases. See Additional file 1 for the full Ovid MEDLINE search.

Database searches were designed to identify primary research studies published in English providing an analysis of an adult (aged $>18$ years) patient sample in an RCT (or number of RCTs or meta-analysis of RCTs) 
compared with an adult patient population treated outside of an RCT setting with the same condition. Studies could have quantitatively assessed how many patients in a realworld population would satisfy the eligibility requirements of an RCT, or compared the clinical characteristics of an RCT sample with a real-world population. Only those studies reporting on pharmaceutical interventions studied as part of an RCT (placebo-controlled or active comparator) were included. Case reports, methodology papers, and conference abstracts were not considered, nor were studies that undertook an analysis of patients who were recruited into an RCT compared with those that declined participation, or studies that involved a pediatric (aged $<18$ years) population. This analysis was limited to studies in cardiology, mental health, and oncology, as the larger numbers of publications identified in these therapeutic areas allowed for a higher level synthesis of their findings.

\section{Study selection, data extraction, and reporting}

Search results were assessed for relevance by two independent researchers by reviewing the title and abstract of all identified studies. Studies meeting or potentially meeting the review eligibility criteria were assessed in more detail using the full text. A third reviewer (TKM) resolved disagreements on study selection.

A data extraction table was developed and tested on a sample of studies before further refinement. Data were quality checked through double-data extraction by a second researcher on $10 \%$ of the records included to ensure the format of data extraction tables was appropriate. All data included in the final manuscript were quality checked. The following data were extracted from each included publication: (i) generalizability objectives; (ii) patient populations and country of study; (iii) methods; (iv) description of RCT and real-world data sources; (v) listing of comparisons made and key results; (vi) overall conclusions; (vii) recommendations addressing identified issues and best practices.

Following a detailed review, a framework for the narrative analysis of the data was developed that included categorization of the identified studies by two methods. Method A involved a formal statistical comparison (for example, use of Wilcoxon rank sum test and chi-square test for continuous and categorical variables, respectively) of baseline characteristics between a real-world patient population and a patient sample enrolled in an RCT in the same specific disease area. Patients were compared for baseline characteristics such as demographics, clinical and disease data, and treatments and procedures. A range of different statistical methodologies were employed in the included studies, and it is outside of the scope of this review to detail them all; the reader is referred to the individual studies for more information. Method B involved a determination of the proportion of patients in a real-world population that would have been trial eligible or ineligible by review of individual patient medical records followed by the application of explicit eligibility criteria derived from specific RCTs or common criteria derived from a review of multiple RCTs in the same disease to individual patient data. The ineligibility rates as calculated in each individual Method B study were tabulated and the distribution by quartiles examined. A minority of studies employed a mixture of methods (A and $B$ ) and presentation of the findings from such studies was split by method (Tables 2 and 3).

In order to interpret the main findings of the literature review as they related to external validity, a qualitative synthesis of individual study results was undertaken. The discussion and conclusions of each publication were closely studied by one researcher, and the subjective author conclusions with respect to "external validity", "generalizability", or "representativeness" were tabulated. These were then grouped according to the precise wording used by individual authors and categorized as: "Different" if the authors explicitly commented that, in their opinion, there were meaningful differences between RCT samples and real-world populations that suggested they were not representative, that the data could not be extrapolated or were not applicable to real-world settings, and/or that external validity is impacted; "Not Explicit" if the authors did not explicitly comment on external validity or did not comment on external validity despite demonstration of differences in baseline characteristics; "Similar" if the authors commented that populations were similar and/or RCT results were generalizable to the overall disease population. A second researcher checked the grouping of each study by category; in the event of any disagreements, the findings of each paper were discussed until resolution was reached.

\section{Results}

\section{Search results}

The study selection is shown in Fig. 1. The original search returned 5,456 studies of which 46 in the areas of cardiology, mental health, and oncology were identified as relevant after abstract review. An additional six studies were identified through citation searching.

\section{Study design}

Of the 52 studies included, 18 (34.6\%) employed only Method A (comparison of baseline characteristics) while 27 (51.9 \%) employed only Method B (determination of percentage ineligibility) (Table 1). An additional seven studies (13.5 \%) used both Methods A and B. The highest number of studies was conducted in the USA (Table 1). The populations studied using Method A were compared for demographics, clinical characteristics, baseline treatments and procedures, and other variables (Table 1). Additional 


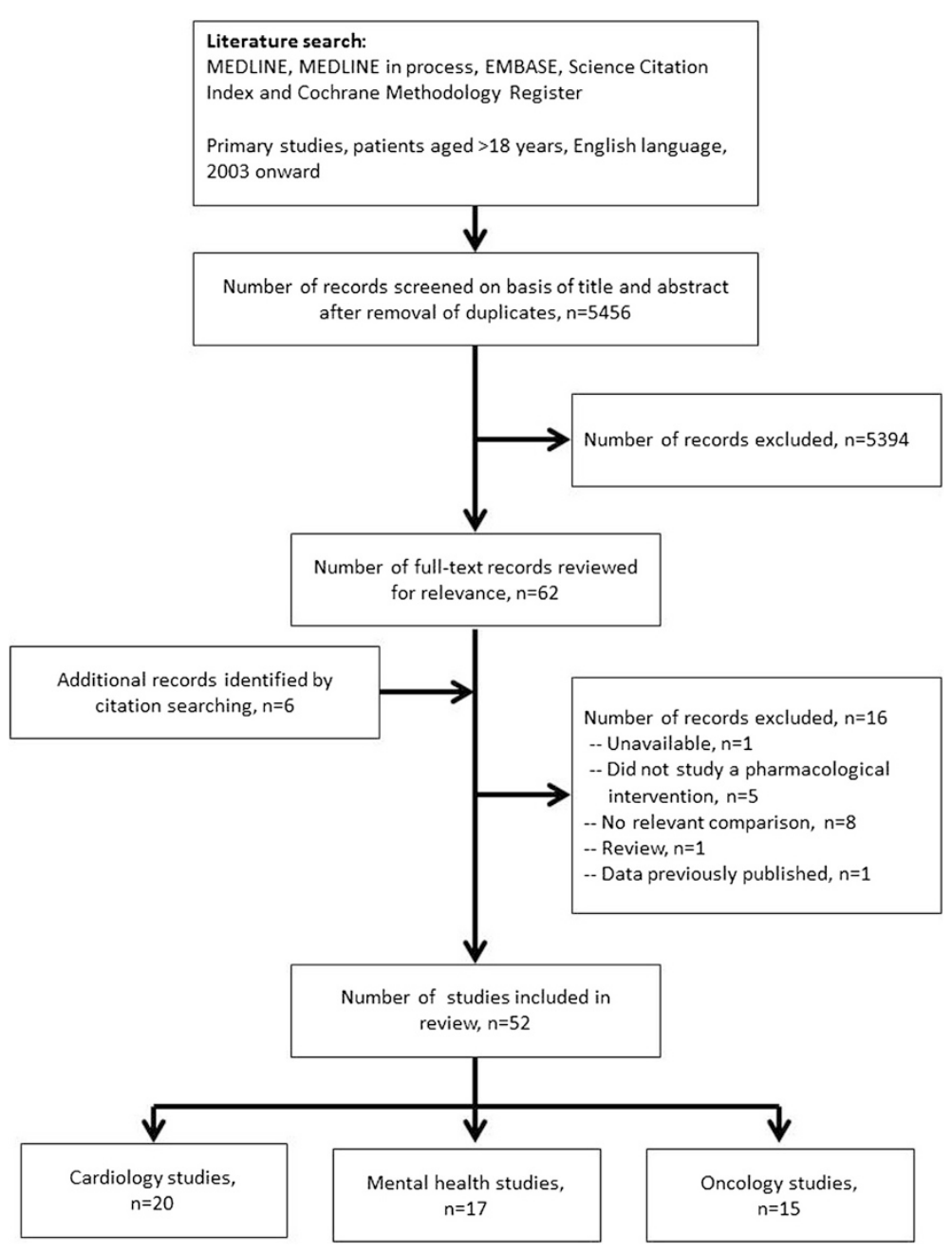

Fig. 1 Study selection for a literature review assessing the external validity of randomized controlled trials

analyses were conducted in some Method B studies as detailed in Table 1 . The sources and settings from which RCT samples and real-world patient populations were drawn are listed in Tables 2 and 3 (a more detailed summary of sources is provided in Additional file 2).

\section{Representativeness/external validity}

In $37(71.2 \%)$ studies (12 [66.7 \%] Method A; 19 [70.3 \%] Method B; 6 [85.7 \%] Method A/B), the individual study authors concluded that RCT samples were not representative of patients encountered in clinical practice and/or that population differences may have a relevant impact on the external validity of the RCT findings [15-51]. The remaining 15 studies [52-66] did not reach an explicit conclusion regarding external validity or concluded that populations were broadly similar, although we note that in some cases the authors still reported differences between RCT samples and real-world populations (Tables 2 and 3) [53, 57, 62, 64, 65].

\section{Cardiology}

Studies included in the review generally demonstrated that, compared with patients enrolled in major cardiology RCTs, patients encountered in everyday practice were more likely to have higher risk characteristics as they were older, more likely to be female and to have clinical impairment and co-morbid disease, were treated less frequently with guideline-recommended therapy, and received fewer in-hospital procedures (Table 2). When RCT inclusion/exclusion criteria were applied to real-world cardiology patients (Method B), those patients who would have been ineligible for RCT participation were more likely to be older and female, to have co-morbid disease, and to less frequently receive guideline-recommended therapy compared with patients who would have been eligible for the trial (Table 3). In 11 studies employing Method B, 18 different sets of eligibility criteria were applied to realworld populations and ineligibility rates reported; in eight cases $(44.4 \%)$ more than $50 \%$ of patients were reported to 
Table 1 Study design overview of included publications

\begin{tabular}{|c|c|c|c|c|}
\hline & \multicolumn{2}{|c|}{ Number (\%) } & \multirow[b]{2}{*}{ Oncology } & \multirow[b]{2}{*}{ Total } \\
\hline & Cardiology & $\begin{array}{l}\text { Mental } \\
\text { health }\end{array}$ & & \\
\hline Total number of studies & $20(38.5)$ & $17(32.7)$ & $15(28.8)$ & $52(100)$ \\
\hline \multicolumn{5}{|l|}{ Geography } \\
\hline USA & $8^{\mathrm{a}}(40.0)$ & $10(58.8)$ & $5(33.3)$ & $23(44.2)$ \\
\hline The Netherlands & $1(5.0)$ & $3(17.6)$ & $2(13.3)$ & $6(11.5)$ \\
\hline Germany & $1(5.0)$ & $2(11.8)$ & $2(13.3)$ & $5(9.6)$ \\
\hline Canada & $3(15.0)$ & $1(5.9)$ & $1(6.7)$ & $5(9.6)$ \\
\hline Other & $7(35.0)$ & $1(5.9)$ & $5(33.3)$ & $13(25.0)$ \\
\hline \multicolumn{5}{|l|}{ Method $^{b}$} \\
\hline A only & $9(45.0)$ & $2(11.8)$ & $7(46.7)$ & $18(34.6)$ \\
\hline B only & $9(45.0)$ & $12(70.6)$ & $6(40.0)$ & $27(51.9)$ \\
\hline$A$ and $B$ & $2(10.0)$ & $3(17.6)$ & $2(13.3)$ & $7(13.5)$ \\
\hline \multicolumn{5}{|c|}{ Comparisons made, Method $A^{c, d}$} \\
\hline Demographics & $10(90.9)$ & $5(100)$ & $8(88.9)$ & $23(92.0)$ \\
\hline Clinical characteristics & $8(72.7)$ & $5(100)$ & $7(77.8)$ & $20(80.0)$ \\
\hline $\begin{array}{l}\text { Treatments and } \\
\text { procedures }\end{array}$ & $4(36.4)$ & $2(40.0)$ & $3(33.3)$ & $9(36.0)$ \\
\hline Other ${ }^{\mathrm{e}}$ & $1(9.1)$ & $1(20.0)$ & $0(0.0)$ & $2(8.0)$ \\
\hline \multicolumn{5}{|c|}{ Additional analyses undertaken, Method B ${ }^{d}$} \\
\hline $\begin{array}{l}\text { Comparison of baseline } \\
\text { characteristics, eligible } \\
\text { vs ineligible patients }\end{array}$ & $6(54.5)$ & $6(40.0)$ & $1(12.5)$ & $13(38.2)$ \\
\hline $\begin{array}{l}\text { Common reasons for } \\
\text { trial ineligibility }\end{array}$ & $7(63.6)$ & $14(93.3)$ & $8(100)$ & $29(85.3)$ \\
\hline
\end{tabular}

${ }^{a}$ Includes one study conducted in the USA and Canada. ${ }^{b}$ Method A, formal statistical comparison of baseline characteristics between a real-world patient population and patients enrolled in a randomized controlled trial (RCT) in the same disease area; Method B, determination of the proportion of real-world patients who would have been trial eligible or ineligible by review of individual patient medical records followed by application of RCT eligibility criteria. ${ }^{C}$ Each study made multiple comparisons. ${ }^{d}$ Percentages calculated based on total number of studies employing method (for example, Method A studies plus Method A/B studies). ${ }^{~}$ Other comparisons included physical activity relative to "others the same age" ( $n=1$ cardiology study) and personality traits ( $n=1$ mental health study)

be ineligible for trial inclusion (Fig. 2 and Table 3). The reasons for ineligibility varied considerably by study depending on the specific condition under assessment.

\section{Mental health}

In general, the identified studies reported that real-world patients with mental health disorders tended to be more severely ill than patients enrolled in RCTs. They also appeared to have more co-morbidities and, in some cases, lower overall functioning and socioeconomic status (Table 2). Studies that assessed the characteristics of a realworld population after the application of specific RCT inclusion/exclusion (Method B) reported that patients who would have been RCT ineligible were older, had more comorbidities and more severe disease, exhibited lower overall functioning, and had lower socioeconomic status than patients who would have been eligible for trial participation (Table 3). In the 15 studies employing Method B, 18 different sets of eligibility criteria were applied to real-world populations resulting in ineligibility rates in excess of $50 \%$ in 16 (88.9 \%) cases (Fig. 2 and Table 3). Common reasons for RCT exclusion across studies included current or history of substance abuse, suicide risk, presence of co-morbidities (such as other Axis I disorder, co-morbid anxiety, and other central nervous system [CNS] or neuromuscular disorder), insufficient symptom duration or low disease severity (in studies of major depressive disease), contraindicated medication, and significant medical condition (see Additional files 3 and 4).

\section{Oncology}

Compared with RCT-enrolled patients, real-world patients with cancer were often older, and more likely to be female, have a poor performance status, and worse disease prognosis (Table 2) in the studies selected in this review. A single study compared the baseline characteristics between RCT ineligible versus eligible patients after the application of inclusion/exclusion criteria and found that ineligible patients with colorectal cancer had a worse performance status (Table 3) [58]. In the eight studies employing Method B, 18 different sets of eligibility criteria were applied to real-world populations, with ineligibility rates greater than $50 \%$ being reported in 12 (66.7 \%) cases (Fig. 2 and Table 3). Reasons for trial exclusion included poor performance status, previous history of cancer, co-morbidities, reduced life expectancy, CNS or brain metastases, and older age (see Additional files 3 and 4).

\section{Potential factors influencing the external validity of RCTs} In the majority of included studies, the authors made some attempt to identify factors influencing the external validity of RCTs. These could broadly be divided into explicit and implicit factors: explicit factors are the inclusion/exclusion criteria listed in the study protocol, while implicit factors include other issues that may affect patient participation in any given trial. The influence of implicit factors on external validity could only be hypothesized in the included studies and are outlined below.

\section{Explicit factors (restrictive inclusion/exclusion criteria)}

Explicit factors were identified as a key driver for differences in RCT samples and real-world populations, as demonstrated by the often high rates of trial ineligibility (Fig. 2 and Table 3) determined in the included studies. By using restrictive inclusion/exclusion criteria, higher risk patients are effectively excluded from RCTs. For example, in cardiology studies, patients often appeared to be excluded on the basis of older age and presence of comorbid disease. The authors of these studies suggested 
Table 2 Key results and main author conclusions from Method A studies

\begin{tabular}{|c|c|c|c|}
\hline Study & Real-world data source & Key differences (real-world versus RCT patients) & $\begin{array}{l}\text { Main author } \\
\text { conclusions }^{\mathrm{b}}\end{array}$ \\
\hline \multicolumn{4}{|l|}{ Cardiology } \\
\hline Badano et al., 2003 [15] & $M C-P R$ & $\begin{array}{l}\text { Older, more female, higher rates of concomitant diabetes, } \\
\text { greater LVF clinical impairment }\end{array}$ & Different \\
\hline Björklund et al., 2004 [17] & MC-PR & Older, more female and more $\mathrm{CV}$ risk factors & Different \\
\hline Costantino et al., $2009^{\mathrm{a}}[21]$ & SC-PR & Older, more female, lower NYHA class & Different \\
\hline Dhruva et al., 2008 [22] & $\mathrm{ID}$ & Older, more female & Different \\
\hline Ezekowitz et al., 2012 [24] & MC-PR & Older, more female, more co-morbidities/prior cancer & Different \\
\hline Golomb et al., 2012 [27] & MC-PR & Increased self-rated physical activity with increasing age & Different \\
\hline Hutchinson-Jaffe et al., 2010 [29] & MC-PR & $\begin{array}{l}\text { Older, more female, more co-morbidities, less guideline- } \\
\text { recommended treatment/procedures }\end{array}$ & Different \\
\hline Melloni et al., 2010 [37] & MC-PR & More female & Different \\
\hline Steinberg et al., 2007 [62] & MC-PR & Older, more co-morbidities/CVD history & NE \\
\hline Uijen et al., 2007ª [44] & MC-PR & Older, more female, higher CVD risk & Different \\
\hline Wagner et al., 2011 [65] & ID & Older, more chronic diseases & NE \\
\hline \multicolumn{4}{|l|}{ Mental health } \\
\hline Kushner et al., 2009 [57] & MC-PR & $\begin{array}{l}\text { Greater depression severity (some scales), lower preference } \\
\text { for novel experiences }\end{array}$ & NE \\
\hline Rabinowitz et al., 2003ª [59] & MC-PR & No major differences & Similar \\
\hline Riedel et al., 2005 [60] & SC-PR & $\begin{array}{l}\text { Older, longer duration of illness, more internistic co-morbidities/ } \\
\text { hospitalizations }\end{array}$ & Similar \\
\hline Surman et al., 2010 & SC-PR & $\begin{array}{l}\text { More co-morbidities, anxiety/depression, alcohol/substance } \\
\text { dependence }\end{array}$ & Different \\
\hline Zarin et al., 2005 [49] & MC-PR & Older, more female/Caucasian & Different \\
\hline \multicolumn{4}{|l|}{ Oncology } \\
\hline Baquet et al., 2009 [52] & $M C-P R$ & $\begin{array}{l}\text { Fewer females (non-sex-specific tumor RCTs), fewer males } \\
\text { (sex-specific tumor RCTs) }\end{array}$ & $\mathrm{NE}$ \\
\hline Elting et al., 2006 [23] & SC-PR & $\begin{array}{l}\text { Older, more females/chronic co-morbidities, worse health/performance } \\
\text { status }\end{array}$ & Different \\
\hline Fraser et al., 2011 [a [25] & MC-PR & $\begin{array}{l}\text { Worse disease prognosis, more drug-related toxicity, lower } \\
\text { drug dose intensity }\end{array}$ & Different \\
\hline Jennens et al., 2006 [30] & MC-PR & Older & Different \\
\hline Kalata et al., 2009 [31] & MC-PR & Older, more females, worse prognosis & Different \\
\hline Mengis et al., 2003ª [38] & SC-PR & $\begin{array}{l}\text { Older, worse performance status, more infections/AML-MDS } \\
\text { subtypes }\end{array}$ & Different \\
\hline van der Linden et al., 2014 [45] & MC-PR & Older, more females, poor prognostic factors & Different \\
\hline Yennurajalingam et al., 2013 [48] & SC-PR & Older, more males, higher symptom intensity scores & Different \\
\hline Yessaian et al., 2005 [66] & MC-PR & No major differences & Similar \\
\hline
\end{tabular}

that cardiovascular disease may represent a more complicated syndrome in such patients [15] and that they are more likely to experience adverse events $[16,19]$. As such, the results from these studies may not provide a complete picture of anticipated drug efficacy and safety in clinical practice. Female patients were also under- 
Table 3 Key results and main author conclusions from Method B studies

\begin{tabular}{|c|c|c|c|c|}
\hline Study & $\begin{array}{l}\text { Real-world data } \\
\text { source }\end{array}$ & $\%$ ineligibility $^{a}$ & Key differences (ineligible versus eligible patients) & $\begin{array}{l}\text { Main authe } \\
\text { conclusion }\end{array}$ \\
\hline \multicolumn{5}{|l|}{ Cardiology } \\
\hline Bahit et al., 2003 [16] & $M C-P R$ & 33.6 & $\begin{array}{l}\text { Older, more females/previous MI, lower ASA use, } \\
\text { longer LOS }\end{array}$ & Different \\
\hline Bosch et al., 2008 [19] & SC-PR & 41.2 & Older, higher risk profile & Different \\
\hline Collet et al., 2003 [53] & SC-PR & 34.0 & $\begin{array}{l}\text { Older, more females, higher risk score, fewer } \\
\text { in-hospital procedures }\end{array}$ & $\mathrm{NE}$ \\
\hline Costantino et al., $2009^{c}[21]$ & SC-PR & 66.2 & ND & Different \\
\hline Fortin et al., 2006 [55] & MC-PR & $1.4-65.5$ & ND & $\mathrm{NE}$ \\
\hline Koeth et al., 2009 [34] & MC-PR & 46.4 & $\begin{array}{l}\text { Older, more females, more diabetes/hypertension, } \\
\text { less guideline-recommended treatment }\end{array}$ & Different \\
\hline \multirow[t]{2}{*}{ Krumholz et al., 2003 [56] } & \multirow[t]{2}{*}{ MC-PR } & 84.5 (NRMI) & \multirow[t]{2}{*}{ ND } & \multirow[t]{2}{*}{ Similar } \\
\hline & & $90.6(C C P)$ & & \\
\hline Lenzen et al., 2005 [35] & MC-PR & 61.6 & $\begin{array}{l}\text { Older, more females, more co-morbid hypertension/ } \\
\text { ACS/renal impairment, less guideline-recommended } \\
\text { treatment at baseline }\end{array}$ & Different \\
\hline Masoudi et al., 2003 [36] & ID & 67.0 & ND & Different \\
\hline Steg et al., 2007 [40] & MC-PR & 33.6 & $\begin{array}{l}\text { Older, history of MI, diabetes, TIA, PAD, and CABG, } \\
\text { less guideline-recommended treatment/procedures, } \\
\text { high risk score }\end{array}$ & Different \\
\hline Uijen et al., $2007^{\complement}$ [44] & MC-PR & 53.0 & ND & Different \\
\hline \multicolumn{5}{|l|}{ Mental health } \\
\hline Blanco et al., 2008 [18] & GP & 75.8 & ND & Different \\
\hline Goedhard et al., 2010 [26] & SC-PR & 69.8 & Older, more Axis II personality disorders & Different \\
\hline \multirow[t]{2}{*}{ Hoertel et al., 2013 [28] } & \multirow[t]{2}{*}{ GP } & 58.2 (bipolar) & \multirow[t]{2}{*}{ ND } & \multirow[t]{2}{*}{ Different } \\
\hline & & 55.8 (mania) & & \\
\hline Keitner et al., 2003 [32] & SC-PR & 85.5 & ND & Different \\
\hline Khan et al., 2005 [33] & GP & 98.2 & ND & Different \\
\hline Rabinowitz et al., 2003 [59] & MC-PR & 33.0 & ND & Similar \\
\hline Seemuller et al., 2010 [61] & MC-PR & 69.0 & Younger, trend to younger age at disease onset & Similar \\
\hline Storosum et al., 2004 [41] & SC-PR & $83.8^{\mathrm{d}}$ & ND & Different \\
\hline Surman et al., $2010^{c}$ [42] & SC-PR & 61.0 & $\begin{array}{l}\text { More lifetime co-morbidity, lower overall functioning/ } \\
\text { SES }\end{array}$ & Different \\
\hline Talamo et al., 2008 [63] & SC-PR & 77.6 & Few differences & Similar \\
\hline van der Lem et al., 2011 [64] & SC-PR & $75.5-81.2^{\mathrm{e}}$ & ND & $\mathrm{NE}$ \\
\hline Wisniewski et al., 2009 [47] & MC-PR & 77.8 & $\begin{array}{l}\text { Older, less educated, more black/Hispanic, longer } \\
\text { disease duration, history of suicide and substance } \\
\text { abuse, more atypical features }\end{array}$ & Different \\
\hline Zarin et al., $2005^{\complement}[49]$ & MC-PR & $\begin{array}{l}55.0 \text { (bipolar) } 38.0 \\
\text { (schizo-phrenia) }\end{array}$ & $\begin{array}{l}\text { More co-morbidity, lower global functioning, greater } \\
\text { use of antipsychotic medication }\end{array}$ & Different \\
\hline Zetin and Hoepner, 2007 [50] & SC-PR & 91.4 & ND & Different \\
\hline Zimmerman et al., 2004 [51] & SC-PR & 65.8 & ND & Different \\
\hline Oncology & & & & \\
\hline Clarey et al., 2012 [20] & SC-PR & $31.0-76.0$ & ND & Different \\
\hline Filion et al., 2012 [54] & SC-PR & $-{ }^{f}$ & ND & Similar \\
\hline Fraser et al., $2011^{\complement}[25]$ & $M C-P R$ & 14.9 & ND & Different \\
\hline Mengis et al., 2003 [38] & SC-PR & 87.0 & ND & Different \\
\hline
\end{tabular}


Table 3 Key results and main author conclusions from Method B studies (Continued)

\begin{tabular}{lllll}
\hline Mol et al., 2013 [58] & MC-PR & 21.5 & $\begin{array}{l}\text { Worse performance status, higher alkaline phosphatase, } \\
\text { less primary tumor resection }\end{array}$ & Similar \\
Somer et al., 2008 [39] & SC-PR & 71.0 & ND & Different \\
Terschüren et al., 2010 [43] & MC-PR & $35.9(\mathrm{HL})$ & ND & Different \\
& & $70.4(\mathrm{hgNHL})$ & & Different \\
\hline
\end{tabular}

Please see Additional files 2 and 4 for more detailed results

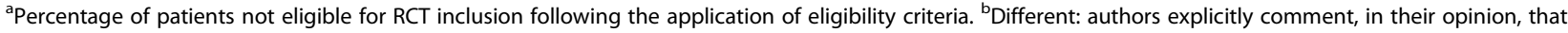
there were meaningful differences between populations that suggested they were not representative, that the data could not be extrapolated or were not applicable to real-world settings, and/or that external validity is impacted; NE: authors do not explicitly comment on external validity or do not comment on external validity despite demonstration of differences in baseline characteristics; Similar: authors comment that populations are similar and/or that RCT results are generalizable to the overall disease population. 'Studies that employed Methods A and B; in these studies RCT samples and real-world populations were compared, the authors then used the eligibility criteria from the RCT of interest to determine how many patients would hypothetically have been eligible or ineligible for that trial. Results presented in this table are for Method B only (see Table 2 for Method A results). ${ }^{d}$ Percentage of manic episodes not number of ineligible. ${ }^{e} 75.5 \%$ based on application of stringent criteria using the Mittman regression equation to calculate HAM-D; $81.2 \%$ based on application of stringent criteria using the Hawley or Zimmerman regression equation to calculate HAM-D. Inclusion/exclusion criteria were categorized to identify criteria that might impede RCT recruitment; if any individual category was not met by $>10 \%$ of patients with breast cancer from a retrospective cohort, then the criterion was considered a barrier to recruitment. ACS acute coronary syndrome, ASA aspirin, CABG coronary artery bypass graft, CCP cooperative cardiovascular project, GP general population data, $H L$ Hodgkin's lymphoma, hgNHL high-grade non-Hodgkin's lymphoma, ID insurance data, LOS length of stay, MC-PR patient records - multicenter (including multicenter registries and observational studies), MI myocardial infarction, ND not determined, NRMI National Registry of Myocardial Infarction, $P A D$ peripheral arterial disease, SC-PR patient records - single center, SES socioeconomic status, TIA transient ischemic attack

represented in the cardiology trials identified in this review $[15,17,24,29,37]$; one of the reasons for this may be due to cardiovascular disease affecting women later in life, meaning that upper age limit restrictions may disproportionately limit their inclusion in RCTs relative to men [37]. In mental health studies, high proportions of patients were excluded on the basis of substance abuse, which is a particular issue for the external validity of trials in bipolar patients where rates are high [41]. One study applied only the

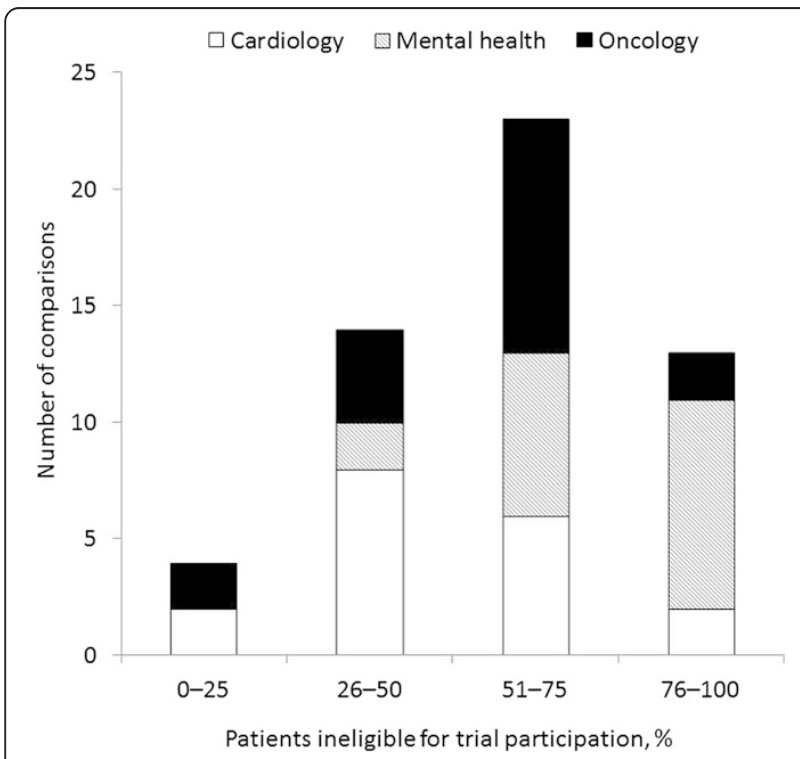

Fig. 2 Proportion of real-world patients ineligible in randomized controlled trials (RCTs) after application of inclusion/exclusion criteria. Method B studies. Some individual studies reported multiple ineligibility rates derived from the application of selection criteria from a number of different RCTs to a single real-world population. Hence, in the 34 studies that employed Method B, 54 different ineligibility rates were calculated exclusion criteria that the authors considered strictly necessary with respect to safety and found that nearly $75 \%$ of patients with depression were still ineligible for participation in efficacy RCTs [50]. Patient samples in oncology trials were often found to have better disease prognosis and better performance status compared with real-world patients with cancer [23, 25, 31, 38, 45]. Inadequate performance status (for example, Eastern Cooperative Oncology Group performance status $\geq 2$ ) was one of the most common reasons for trial exclusion in several studies [20, 39, 46, 58].

\section{Implicit factors}

Implicit factors that may have affected the external validity of RCTs were also identified in some of the studies reviewed. Two cardiology studies noted that issues with informed consent, whereby the most severely ill patients are less likely to give informed consent or it is harder to gain informed consent, may lead to the selection of lower risk patients for trial participation [16, 17]. In addition, one study indicated that psychiatric patients with more severe aggression were also less likely to consent to enter an RCT [26]. The type of RCT setting and/or recruitment method were also discussed as potential barriers to trial participation [26, 33, 49]; for example, one study that evaluated how many patients with schizophrenia would be eligible for antipsychotic clinical trials suggested that there could be discrepancies between subjects who were recruited through advertisement and those recruited in a clinical setting [33]. In oncology patients (and their physicians), one of the biggest barriers to trial participation was noted to be fear of randomization to the placebo arm [43]. A number of other patient-related factors were also identified, including logistical issues related to study participation, beliefs and attitudes regarding the safety 
of trial medications, cultural factors, level of satisfaction with current treatment, and willingness to participate $[39,43,48,49]$. Finally, one study demonstrated that patients who participate in trials may have different personality traits than those who do not; patients with depression who were enrolled in an antidepressant medication RCT were found to score more highly on a personality scale that assessed preferences for novel experiences compared with non-participants [57].

\section{Study recommendations for the improvement of external validity}

Many of the studies included in the present review made recommendations to improve the external validity of RCTs. These recommendations are outlined in Table 4

Table 4 Recommendations for managing external validity issues made by included studies

Patient populations

Broadening of RCT inclusion and exclusion criteria $[19,20,29,31-33,36$, $38,40,42,44,47,49]$

Selection of patients from more appropriate settings/populations to achieve a more representative sample (for example, prospective use of registry data; a priori estimation of patient eligibility by application of trial exclusion criteria to the target population) $[15,17,18,31,44,54]$

Conduct of RCTs in specific patient subgroups $[20,28,30,31,46]$

Standardization of inclusion/exclusion criteria and diagnostic and screening assessments across RCTs in a given medical condition [51]

Intervention

Broader range of RCT treatments (that is, different and realistic dosing regimens, use of concurrent therapy, and appropriate duration of treatment); comparison of new treatments with treatments as usual rather than to a prescribed dose of a particular medicine [49]

Reporting

Improved reporting of populations and results (that is, greater transparency in the reporting of how exclusion criteria are operationalized and how this influences eligibility, and of the rate and major characteristics of excluded patients) $[28,38,51]$

Collection, reporting, and comparison of data from patients within and outside of the trial $[24,28,63]$

Analysis

Development of statistical analysis plans and power calculation adjustment to ensure adequate powering for subgroup analyses [20,37]

Generation of supportive data

Conduct of observational studies after the demonstration of treatment efficacy at the RCT level $[15,23,36]$

Development of large patient registries in specific disease areas [19]

Adoption of pragmatic studies $[48,49]$

Clinical practice recommendations

Prospective auditing of drug efficacy and safety in everyday practice settings and comparison of these data with RCT results [25]

Provision of more detailed product information to include the criteria by which patients were selected in pivotal RCTs [20]

$R C T$ randomized controlled trial and include modifying RCT design to improve external validity directly, and generating complementary evidence from alternative study types to address the limited external validity of the RCT post hoc.

\section{Discussion}

The present analysis utilized a robust literature review methodology to identify studies that compared the clinical characteristics of an RCT sample and patients from a realworld source (Method A) or assessed the proportion of a real-world population that would satisfy criteria for RCT inclusion (Method B). Publications identified by this methodology indicated that RCT samples in cardiology, mental health, and oncology studies that assessed pharmaceutical interventions in adult patients were often not broadly representative of patients treated in everyday clinical practice and that caution should be exercised when extrapolating data from trials to patients treated in usual care settings. Note that, with the exception of a single study [40], none of the RCTs described in the included studies were documented as being of a pragmatic design. In this Method B study, the RCTs in acute coronary syndrome from which eligibility criteria were extracted were described as having pragmatic enrollment strategies; however, the analysis still suggested that there were important differences in risk profile between RCT eligible and ineligible patients [40]. Differences in demographics, clinical characteristics, and treatments and procedures were reported between RCT and real-world patients by studies that employed Method A in their analyses $[15,17,21-25,27,29-31,37,38,42$, $44,45,48,49]$. Similarly, when specific RCT inclusion/ exclusion criteria were applied to real-world populations (Method B), important differences with respect to demographics and clinical and treatment parameters were identified between patients who would have been RCT ineligible compared with those who would have been eligible for the trial $[16,18-21,25,26,28,32-36,38-44,46$, $47,49-51]$. Furthermore, it was observed that large proportions of the general disease population were often excluded from trial participation. We note that some differences in generalizability were observed between the different therapeutic areas studied in the present review.

In only a minority of studies did the authors conclude that RCT samples were broadly representative of realworld populations and that external validity was not impacted, or failed to reach an explicit conclusion regarding external validity despite demonstrating some differences in baseline characteristics between groups [52-66]. These findings are largely consistent with a previously published systematic sampling review that assessed the nature and extent of exclusion criteria among RCTs published between 1994 and 2006 in selected medical journals with impact factors $>2.5$ [2]. While involving the review of older studies and use of more restrictive search criteria 
than the present review, this earlier study also demonstrated that RCTs often exclude large proportions of the general disease population and specific patient groups from trial participation. In agreement with the present review, it was reported that the elderly, women, and patients with co-morbidities were frequently ineligible for trial inclusion [2]. However, note that RCT findings may still be externally valid even in circumstances where the patient sample is not broadly representative of the real-world population. For example, one study included in the present review concluded that patients with unstable angina or non-ST-segment elevation myocardial infarction who would have been excluded from enoxaparin RCTs could be safety treated in clinical practice [53].

That the external validity of RCT results is often limited is widely acknowledged by clinicians as a problem when it comes to extrapolating data to the patients seen in everyday practice $[3,7]$. Indeed, it is an often-cited reason for the frequent underuse of guideline-recommended therapies [67]. Where there is no evidence of efficacy in specific patient groups, clinicians may well be right in withholding treatment so as to prevent unanticipated harm [35]. This situation could, however, mean that patients at highest baseline risk who might be expected to receive the most benefit from a particular therapy are undertreated. This so-called "treatment-risk paradox" has been well described, particularly in cardiology [6].

In the studies included in the present review, the use of restrictive inclusion/exclusion criteria in RCTs was identified as being one of the key factors that limited the external validity of trial findings. Authors reported that frequently excluded patients were the elderly, females, or those with co-morbidities in cardiology studies [15-17, $19,24,29,34,35,40,44,53,55]$, patients with evidence of substance abuse or co-morbid psychological disorders in mental health studies $[18,28,32,33,41,42,47,49,50,61$, 64], and patients with poor disease prognosis in oncology studies [20, 25, 31, 38, 39, 45, 46]. These RCT populations were, therefore, often highly selected and represented a patient sample at much lower risk of adverse events and complications compared with patients in clinical practice. The use of stringent selection criteria in RCTs ensures a homogeneous patient sample, optimizes internal validity of the study by reducing variance and removing potential confounding, so increasing the likelihood of finding a true association between treatment exposure and outcomes (that is, it makes it easier to distinguish the "signal" [treatment effect] from the "noise" [bias and chance]) $[68,69]$. While the use of highly selected populations does not necessarily imply that a given treatment under study would fail to have equivalent efficacy and safety in under-represented patient groups, it does create uncertainty that can only be dispelled through the generation of additional evidence. However, it is pertinent to also consider how inclusion of high-risk patients may affect the outcomes of traditional trials. Patients with more co-morbidities or co-interventions may be more likely to prematurely discontinue study participation, which could lead to high attrition rates and a negative impact on trial validity and outcomes.

The studies reviewed herein made several recommendations to either improve the external validity of RCTs or compensate for limitations thereof. These included adaptation of trial designs to include a more heterogeneous patient sample that better represents different subgroups such as the elderly or patients with comorbidities [19, 20, 28-33, 46]. Some studies suggested that adoption of pragmatic trial designs may be a way forward $[48,49]$. Traditional RCTs are often described as "explanatory" trials since they aim to evaluate treatment efficacy under idealized conditions, and to explore "if and how an intervention works". In contrast, pragmatic trials evaluate the effects of an intervention under usual conditions and their designs seek to determine "if an intervention actually works in real-life" [70]. In recent years, the Pragmatic-Explanatory Continuum Indicator Summary (PRECIS) tool has been developed, and has now been updated with the PRECIS-2 version to allow trialists to design studies that better support the needs of the intended users of the results. PRECIS-2 consists of nine domains (including "participant eligibility criteria") in which design decisions are made to determine the extent to which the trial is pragmatic or explanatory, and to help ensure that the design achieves the primary purpose of the trial [71]. In addition to its application as an aid to trial design, PRECIS-2 has the potential for use in the assessment of completed trials for methodological quality and the likelihood of outcome bias in much the same way as the current Grading of Research, Assessment, Development and Evaluation (GRADE) system is used to assist guideline developers.

There is growing interest in different analytical methods that utilize data from multiple studies to extend and complement the evidence provided by a single clinical trial. Meta-analysis [72, 73] can be used to combine evidence from multiple clinical trials to provide a more valid estimate of treatment effect, assuming the studies being combined are similar enough to permit synthesis. Cross-design synthesis is a type of meta-analysis in which evidence from studies with complementary designs are combined in an effort to leverage complementary strengths (such as internal validity of RCTs and external validity of observational studies) and minimize the weaknesses of each [74]. Another approach that leverages real-world data to extend findings from a traditional trial involves development of propensity scores that predict, for each trial subject, membership in a corresponding real-world population $[75,76]$. Subjects over-represented in the clinical 
trial relative to the target real-world population receive lower weights while those under-represented receive higher weights. The resulting weights can be used to understand differences between the trial and target realworld populations, and to "project" the RCT efficacy to the target population, in effect providing an estimate of the efficacy that would be observed were the trial to be conducted in a more representative everyday practice population $[75,76]$. Finally, simple descriptive analysis of real-world data can also be employed in the trial planning stages to better understand the impact of specific design decisions (for example, potential exclusion criteria) on the anticipated generalizability of the trial results and so improve design. Adaptation of statistical analysis plans was recommended by two of the studies reviewed here as a method to facilitate analysis of important patient subgroups [20,37].

Several of the reviewed studies highlighted incomplete reporting as a potential issue for the external validity of RCTs $[24,28,38,51,63]$. Improvements in trial reporting to provide a more detailed description of RCT samples would enable clinicians to better assess the external validity of RCTs and so more accurately extrapolate trial findings to their own patients. Following reporting guidelines such as CONSORT, which is a requirement for publication in many peer-reviewed journals [1], may go some way to address issues of inconsistent reporting and may provide greater transparency with respect to trial eligibility.

Trials should follow the need for evidence but be part of a broader strategy for evidence generation. As such, complementary data obtained from other appropriately designed alternatives conducted in Phase IV of the development lifecycle are required to address limitations in the external validity of RCTs post hoc. As recommended by some of the studies included in this review $[15,23,36]$, the use of non-randomized observational studies that utilize large healthcare databases can support RCT findings by determining treatment effectiveness in routine clinical practice $[6,77]$. Such studies include a wide range of different designs including prospective and retrospective cohort studies, case-control studies, and cross-sectional studies in which any intervention studied is determined by clinical practice and not a rigid protocol [78]. Taken together, RCT and observational study data should provide a complementary body of evidence that optimizes both internal and external validity.

The findings presented in this review must be viewed within the limitations of the methodology employed. Firstly, the search strategy did not define the outcomes to be reported a priori and was influenced by the evidence base identified. Secondly, there are no acknowledged methods for the assessment of the quality of data for this type of analysis. Thirdly, the present review was limited to just three therapeutic areas (cardiology, mental health, and oncology), and while a large proportion of the relevant literature was focused in these areas, it is possible that findings may be different in other specialties. In addition, to manage the scope of the review, we restricted our eligibility criteria to studies that included adults and assessed pharmaceutical interventions only, and we cannot completely rule out the possibility that findings might be different in pediatric populations or other healthcare interventions. Finally, the conclusions regarding external validity, as reported in individual studies, were subjective, which limited our ability to more accurately synthesize and summarize the findings. The review strategy was, however, relevant to the objective of the present analysis, as it utilized a robust and transparent approach in order to identify key concepts and the main sources of information available on the representativeness of RCT patient samples and the external validity of RCT findings. The framework for categorizing the methods used in individual studies and for interpreting individual study conclusions was consistent and clearly detailed, adding to the methodological rigor of the review.

\section{Conclusions}

In the majority of studies included in this literature review it was concluded that patient samples in cardiology, mental health, and oncology RCTs are not broadly representative of patients encountered in everyday practice. These findings suggest that, while explanatory RCTs still represent the gold-standard primary study design for the generation of clinical efficacy evidence, there is a need to improve their external validity and/or supplement their results with data from a range of research approaches such that physicians treating patients in real-world settings have the appropriate evidence on which to base their clinical decisions and to provide greater insight regarding clinical effectiveness in everyday practice. This goal could be achieved in two ways: (i) modification of trial designs to include a patient sample more representative of the individuals expected to receive an intervention in real life, while recognizing the potential compromise of internal validity caused by increasing heterogeneity as discussed above [68, 69]; and (ii) supplementing RCT evidence with data generated from a continuum of appropriately designed supportive studies with alternative methodologies. In general, a thoughtful approach to RCT design is required in which the tradeoffs between internal and external validity are considered in a holistic and balanced manner so that the results can better meet the diverse needs of regulators, prescribers, payers, and patients. 


\section{Additional files}

\section{Additional file 1: Full Ovid MEDLINE search strategy for literature} searches. (PDF $280 \mathrm{~kb}$ )

Additional file 2: Summary of real-world and RCT data sources employed in included studies. Detailed description of data sources (real-world and RCT) used in studies included in review. (PDF $207 \mathrm{~kb}$ )

Additional file 3: Key results and author conclusions from studies that compared baseline characteristics between a real-world patient population and a patient sample enrolled in an RCT (Method A). Detailed description of results and subjective author conclusions from studies included in the review that employed Method A. (PDF $207 \mathrm{~kb}$ )

Additional file 4: Key results and main author conclusions from studies assessing rates of ineligibility for RCT participation in a real-world patient population (Method B). Detailed description of results and subjective author conclusions from studies included in the review that employed Method B. (PDF 227 kb)

\section{Abbreviations}

CNS: central nervous system; PRECIS: Pragmatic-Explanatory Continuum Indicator Summary; RCT: randomized controlled trial.

\section{Competing interests}

SC, DF, and JJ are employees of Eli Lilly and Company, USA. TKM is Director of, and SR is an employee of, Kennedy-Martin Health Outcomes Ltd, and received financial support from Eli Lilly and Company for their contributions to the conception and design of the study; the acquisition, analysis, and interpretation of the data; and drafting of the manuscript.

\section{Authors' contributions}

SC, DF, and JJ conceived the project. TKM conducted the literature search. TKM and SR reviewed the search results and conducted the data extraction. All authors contributed to the content and writing of the manuscript and all authors read and approved the final manuscript.

\section{Acknowledgements}

This study was supported by Eli Lilly and Company, USA. The authors thank Mick Arber for his assistance with the literature review.

\section{Author details}

${ }^{1}$ Kennedy-Martin Health Outcomes Ltd, 3rd Floor, Queensberry House, 106 Queens Road, Brighton BN1 3XF, UK. ${ }^{2}$ Eli Lilly and Company, Indianapolis, Indiana, USA.

Received: 9 March 2015 Accepted: 21 October 2015

Published online: 03 November 2015

\section{References}

1. Schulz KF, Altman DG, Moher D, for the CONSORT Group. CONSORT 2010 Statement: Updated guidelines for reporting parallel group randomized trials. Ann Intern Med. 2010;152:726-32.

2. Van Spall HG, Toren A, Kiss A, Fowler RA, et al. Eligibility criteria of randomized controlled trials published in high-impact general medical journals: a systematic sampling review. JAMA. 2007:297:1233-40.

3. Rothwell PM. External validity of randomised controlled trials: "to whom do the results of this trial apply?". Lancet. 2005;365:82-93.

4. Singal AG, Higgins PDR, Waljee AK. A primer on effectiveness and efficacy trials. Clin Trans Gastroenterol. 2014:5:e45.

5. Rothwell PM. Factors that can affect the external validity of randomised controlled trials. PLoS Clin Trials. 2006;1:e9.

6. Nallamothu BK, Hayward RA, Bates ER. Beyond the randomized clinical trial: the role of effectiveness studies in evaluating cardiovascular therapies. Circulation. 2008:118:1294-303.

7. Sniderman AD, LaChapelle K, Rachon NA, Furberg CD, et al. The necessity for clinical reasoning in the era of evidence-based medicine. Mayo Clin Proc. 2013;88:1108-14.

8. Franciosa JA. The potential role of community-based registries to complement the limited applicability of clinical trial results to the community setting: heart failure as an example. Am J Manag Care. 2004:10:487-92.

9. Saunders C, Byrne CD, Guthrie B, Lindsay RS, McKnight JA, Sattar N, et al. External validity of randomized controlled trials of glycaemic control and vascular disease: how representative are participants? Diabet Med. 2013;30:300-8

10. Hordijk-Trion M, Lenzen M, Wijns W, De Jagere P, Simmons ML, Scholte Op Reimer WJM, et al. Patients enrolled in coronary intervention trials are not representative of patients in clinical practice: results from the Euro Heart Survey on Coronary Revascularization. Eur Heart J. 2006;27:671-8.

11. Maasland L, Van Oostenbrugge RJ, Franke CF, Scholte Op Reimer WJM, Koudstaal PJ, Dippel DWJ, et al. Patients enrolled in large randomized clinical trials of antiplatelet treatment for prevention after transient ischemic attack or ischemic stroke are not representative of patients in clinical practice: the Netherlands Stroke Survey. Stroke. 2009;40:2662-8.

12. Travers J, Marsh S, Williams M, Weatherall M, Caldwekk B, Shirtcliffe $P$, et al. External validity of randomised controlled trials in asthma: to whom do the results of the trials apply? Thorax. 2007:62:219-23.

13. Villela R, Yuen SY, Pope JE, Baron M. Assessment of unmet needs and the lack of generalizability in the design of randomized controlled trials for scleroderma treatment. Arthritis Rheum. 2008;59:706-13.

14. Arksey H, O'Malley L. Scoping studies: towards a methodological framework. Int J Soc Res Methodol. 2005;8:19-32.

15. Badano LP, Di Lenarda A, Bellotti P, Albanese MC, Sinagra G, Fioretti PM Patients with chronic heart failure encountered in daily clinical practice are different from the "typical" patient enrolled in therapeutic trials. Ital Heart J. 2003:4:84-91.

16. Bahit MC, Cannon CP, Antman EM, Murphy SA, Gibson MC, McCabe CH, et al. Thrombolysis in myocardial infarction. Direct comparison of characteristics, treatment, and outcomes of patients enrolled versus patients not enrolled in a clinical trial at centers participating in the TIMI 9 Trial and TIMI 9 registry. Am Heart J. 2003;145:109-17.

17. Björklund E, Lindahl B, Stenestrand U, Swahn E, Delborg M, Pehrsson K, et al. Outcome of ST-elevation myocardial infarction treated with thrombolysis in the unselected population is vastly different from samples of eligible patients in a large-scale clinical trial. Am Heart J. 2004;148:566-73.

18. Blanco C, Olfson M, Goodwin RD, Ogburn E, Liebowitz MR, Nunes EV, et al. Generalizability of clinical trial results for major depression to community samples: results from the National Epidemiologic Survey on Alcohol and Related Conditions. J Clin Psychiatry. 2008;69:1276-80.

19. Bosch X, Delgado V, Verbal F, Bórquez E, Loma-Osorio P, Díez-Aja S, et al. Causes of ineligibility in randomized controlled trials and long-term mortality in patients with non-ST-segment elevation acute coronary syndromes. Int J Cardiol. 2008;124:86-91.

20. Clarey J, Kao SC, Clarke SJ, Vardy J. The eligibility of advanced non-small-cell lung cancer patients for targeted therapy clinical trials. Ann Oncol. 2012;23:1229-33

21. Costantino G, Rusconi AM, Duca PG, Giorgia Duca P, Guzzetti S, Bossi I, et al. Eligibility criteria in heart failure randomized controlled trials: a gap between evidence and clinical practice. Intern Emerg Med. 2009;4:117-22.

22. Dhruva SS, Redberg RF. Variations between clinical trial participants and Medicare beneficiaries in evidence used for Medicare national coverage decisions. Arch Intern Med. 2008;168:136-40.

23. Elting LS, Cooksley C, Bekele BN, Frumovitz M, Avritscher EBC, Sun C, et al. Generalizability of cancer clinical trial results: prognostic differences between participants and nonparticipants. Cancer. 2006;106:2452-8.

24. Ezekowitz JA, Hu J, Delgado D, Hernandez AF, Kaul P, Leader R, et al. Acute heart failure: perspectives from a randomized trial and a simultaneous registry. Circ Heart Fail. 2012;5:735-41.

25. Fraser J, Steele N, Al Zaman A, Yule A. Are patients in clinical trials representative of the general population? Dose intensity and toxicities associated with FE100C-D chemotherapy in a non-trial population of node positive breast cancer patients compared with PACS-01 trial group. Eur J Cancer. 2011;47:215-20

26. Goedhard LE, Stolker JJ, Nijman HL, Egberts TCG, Heerdink ER. Trials assessing pharmacotherapeutic management of aggression in psychiatric patients: comparability with clinical practice. Pharmacopsychiatry. 2010;43:205-9.

27. Golomb BA, Chan VT, Evans MA, Koperski S, White HL, Criqui MH. The older the better: are elderly study participants more non-representative? A crosssectional analysis of clinical trial and observational study samples. BMJ Open. 2012;2:e000833. 
28. Hoertel N, Le Strat Y, Lavaud P, Dubertret C, Limosin F. Generalizability of clinical trial results for bipolar disorder to community samples: findings from the National Epidemiologic Survey on Alcohol and Related Conditions. J Clin Psychiatry. 2013;74:265-70.

29. Hutchinson-Jaffe AB, Goodman SG, Yan RT, Wald R, Elbarouni B, Rose B, et al. Comparison of baseline characteristics, management and outcome of patients with non-ST-segment elevation acute coronary syndrome in versus not in clinical trials. Am J Cardiol. 2010;106:1389-96.

30. Jennens RR, Giles GG, Fox RM. Increasing underrepresentation of elderly patients with advanced colorectal or non-small-cell lung cancer in chemotherapy trials. Intern Med J. 2006:36:216-20.

31. Kalata P, Martus P, Zettl H, Rödel C, Hohenberger W, Raab R, et al. Differences between clinical trial participants and patients in a populationbased registry: the German Rectal Cancer Study vs the Rostock Cancer Registry. Dis Colon Rectum. 2009;52:425-37.

32. Keitner Gl, Posternak MA, Ryan CE. How many subjects with major depressive disorder meet eligibility requirements of an antidepressant efficacy trial? J Clin Psychiatry. 2003;64:1091-3.

33. Khan AY, Preskorn SH, Baker B. Effect of study criteria on recruitment and generalizability of the results. J Clin Psychopharmacol. 2005;25:271-5.

34. Koeth O, Zahn R, Gitt AK, Bauer T, Juenger C, Senges J, et al. Clinical benefit of early reperfusion therapy in patients with ST-elevation myocardial infarction usually excluded from randomized clinical trials (results from the Maximal Individual Therapy in Acute Myocardial Infarction Plus [MITRA Plus] registry). Am J Cardiol. 2009;104:1074-7.

35. Lenzen MJ, Boersma E, Scholte Op Reimer WJM, Balk AHMM, Komajda M, Swedberg K, et al. Under-utilization of evidence-based drug treatment in patients with heart failure is only partially explained by dissimilarity to patients enrolled in landmark trials: a report from the Euro Heart Survey on Heart Failure. Eur Heart J. 2005:26:2706-13.

36. Masoudi FA, Havranek EP, Wolfe P, Gross CP, Rathore SS, Steiner JF, et al. Most hospitalized older persons do not meet the enrollment criteria for clinical trials in heart failure. Am Heart J. 2003;146:250-7.

37. Melloni C, Berger JS, Wang TY, Gunes F, Stebbins A, Pieper KS, et al. Representation of women in randomized clinical trials of cardiovascular disease prevention. Circ Cardiovasc Qual Outcomes. 2010;3:135-42.

38. Mengis C, Aebi S, Tobler A, Dähler W, Fey MF. Assessment of differences in patient populations selected for excluded from participation in clinical phase III acute myelogenous leukemia trials. J Clin Oncol. 2003;21:3933-9.

39. Somer RA, Sherman E, Langer CJ. Restrictive eligibility limits access to newer therapies in non-small-cell lung cancer: the implications of Eastern Cooperative Oncology Group 4599. Clin Lung Cancer. 2008:9:102-5.

40. Steg PG, López-Sendón J, Lopez De Sa E, Goodman SG, Gore JM, Anderson FA, et al. External validity of clinical trials in acute myocardial infarction. Arch Intern Med. 2007;167:68-73.

41. Storosum JG, Fouwels A, Gispen-de Wied CC, Wohlfarth T, Van Zwieten BJ, van den Brink W. How real are patients in placebo-controlled studies of acute manic episode? Eur Neuropsychopharmacol. 2004;14:319-23.

42. Surman CB, Monuteaux MC, Petty CR, Faraone SV, Spencer TJ, Chu NF, et al. Representativeness of participants in a clinical trial for attention-deficit/ hyperactivity disorder? Comparison with adults from a large observational study. J Clin Psychiatry. 2010;71:1612-6.

43. Terschüren C, Gierer S, Brillant C, Paulus U, Löffler M, Hoffmann W. Are patients with Hodgkin lymphoma and high-grade non-Hodgkin lymphoma in clinical therapy optimization protocols representative of these groups of patients in Germany? Ann Oncol. 2010;21:2045-51.

44. Uijen AA, Bakx JC, Mokkink HG, Van Weel C. Hypertension patients participating in trials differ in many aspects from patients treated in general practices. J Clin Epidemiol. 2007;60:330-5.

45. van der Linden N, Van Gils CW, Pescott CP, Buter J, Uyl-de Groot CA. Cetuximab in locally advanced squamous cell carcinoma of the head and neck: generalizability of EMR 062202-006 trial results. Eur Arch Otorhinolaryngol. 2014;271:1673-8.

46. Vardy J, Dadasovich R, Beale P, Boyer M, Clarke SJ. Eligibility of patients with advanced non-small cell lung cancer for phase III chemotherapy trials. BMC Cancer. 2009:9:130.

47. Wisniewski SR, Rush AJ, Nierenberg AA, Gaynes BN, Warden D, Luther JF, et al. Can phase III trial results of antidepressant medications be generalized to clinical practice? A STAR*D report. Am J Psychiatry. 2009;166:599-607.

48. Yennurajalingam S, Kang JH, Cheng HY, Chisholm GB, Kwon JH, Palla SL, et al. Characteristics of advanced cancer patients with cancer-related fatigue enrolled in clinical trials and patients referred to outpatient palliative care clinics. J Pain Symptom Manage. 2013;45:534-41.

49. Zarin DA, Young JL, West JC. Challenges to evidence-based medicine: a comparison of patients and treatments in randomized controlled trials with patients and treatments in a practice research network. Soc Psychiatry Psychiatr Epidemiol. 2005:40:27-35.

50. Zetin M, Hoepner CT. Relevance of exclusion criteria in antidepressant clinical trials: a replication study. J Clin Psychopharmacol. 2007;27:295-301.

51. Zimmerman M, Chelminski I, Posternak MA. Exclusion criteria used in antidepressant efficacy trials: consistency across studies and representativeness of samples included. J Nerv Ment Dis. 2004;192:87-94.

52. Baquet CR, Ellison GL, Mishra SI. Analysis of Maryland cancer patient participation in National Cancer Institute-supported cancer treatment clinical trials. J Health Care Poor Underserved. 2009;20(2 Suppl):120-34.

53. Collet JP, Montalescot G, Fine E, Golmard J-L, Dalby M, Choussat R, et al. Enoxaparin in unstable angina patients who would have been excluded from randomized pivotal trials. J Am Coll Cardiol. 2003:41:8-14.

54. Filion M, Forget G, Brochu O, Provencher L, Desbien SC, Doyle C, et al. Eligibility criteria in randomized phase II and III adjuvant and neoadjuvant breast cancer trials: not a significant barrier to enrollment. Clin Trials. 2012;9:652-9.

55. Fortin M, Dionne J, Pinho G, Gignac J, Almirall J, Lapointe L. Randomized controlled trials: do they have external validity for patients with multiple comorbidities? Ann Fam Med. 2006;4:104-8.

56. Krumholz HM, Gross CP, Peterson ED, Barron HV, Radford MJ, Parsons LS, et al. Is there evidence of implicit exclusion criteria for elderly subjects in randomized trials? Evidence from the GUSTO-1 study. Am Heart J. 2003;146:839-47.

57. Kushner SC, Quilty LC, McBride C, Bagby RM. A comparison of depressed patients in randomized vs nonrandomized trials of antidepressant medication and psychotherapy. Depress Anxiety. 2009;26:666-73.

58. Mol L, Koopman M, Van Gils CW, Ottevanger PB, Punt CJA. Comparison of treatment outcome in metastatic colorectal cancer patients included in a clinical trial versus daily practice in The Netherlands. Acta Oncol. 2013;52:950-5.

59. Rabinowitz J, Bromet EJ, Davidson M. Are patients enrolled in first episode psychosis drug trials representative of patients treated in routine clinical practice? Schizophr Res. 2003;61:149-55.

60. Riedel M, Strassnig M, Müller N, Zwack P, Möller H-J. How representative of everyday clinical populations are schizophrenia patients enrolled in clinical trials? Eur Arch Psychiatry Clin Neurosci. 2005;255:143-8.

61. Seemüller F, Möller HJ, Obermeier M, Adli M, Bauer M, Kronmüller K, et al. Do efficacy and effectiveness samples differ in antidepressant treatment outcome? An analysis of eligibility criteria in randomized controlled trials. J Clin Psychiatry. 2010;71:1425-33.

62. Steinberg BA, Moghbeli N, Buros J, Ruda M, Parkhomenko A, Raju BS, et al. Global outcomes of ST-elevation myocardial infarction: comparisons of the Enoxaparin and Thrombolysis Reperfusion for Acute Myocardial Infarction Treatment-Thrombolysis In Myocardial Infarction study 25 (EXTRACT-TIMI 25) registry and trial. Am Heart J. 2007;154:54-61.

63. Talamo A, Baldessarini RJ, Centorrino F. Comparison of mania patients suitable for treatment trials vs clinical treatment. Hum Psychopharmacol. 2008;23:447-54

64. van der Lem R, van der Wee NJ, Van Veen T, Zitman FG. The generalizability of antidepressant efficacy trials to routine psychiatric out-patient practice. Psychol Med. 2011:41:1353-63.

65. Wagner TH, Holman W, Lee K, Sethi G, Ananth L, Thai H, et al. The generalizability of participants in Veterans Affairs Cooperative Studies Program 474, a multi-site randomized cardiac bypass surgery trial. Contemp Clin Trials. 2011;32:260-6.

66. Yessaian A, Mendivil AA, Brewster WR. Population characteristics in cervical cancer trials: search for external validity. Am J Obstet Gynecol. 2005;192:407-13

67. Garfield FB, Garfield JM. Clinical judgment and clinical practice guidelines. Int J Technol Assess Health Care. 2000;16:1050-60.

68. Velasco E. Inclusion criteria. In: Salkind NJ, editor. Encyclopedia of research, volume 1. Thousand Oaks: SAGE Publications, Inc; 2010. p. 589-91.

69. Fletcher R, Fletcher SW, Fletcher GS. Chapter 9, Treatment. In: Fletcher R, Fletcher SW, Fletcher GS, editors. Clinical epidemiology: the essentials. 5th ed. Baltimore: Wolters Kluwer; 2014. p. 132-52.

70. Patsopoulos NA. A pragmatic view on pragmatic trials. Dialogues Clin Neurosci. 2011;13:217-24. 
71. Loudon K, Treweek S, Sullivan F, Donnan P, Thorpe KE. The PRECIS-2 tool: designing tools that are fit for purpose. BMJ. 2015;350:h2147.

72. Sutton AJ, Higgins JP. Recent developments in meta-analysis. Stat Med. 2008;27:625-50

73. Prevost TC, Abrams KR, Jones DR. Hierarchical models in generalized synthesis of evidence: an example based on studies of breast cancer screening. Stat Med. 2000;19:3359-76.

74. United States General Accounting Office. Cross design synthesis. A new strategy for medical effectiveness research. United States Government. 1992. http://www.gao.gov/assets/160/151472.pdf. Accessed 2 Jul 2015.

75. Stuart EA, Cole SR, Bradshaw CP, Leaf PJ. The use of propensity scores to assess the generalizability of results from randomized trials. JR Statist Soc A. 2011;174:369-86.

76. Pressler TR, Kaizar EE. The use of propensity scores and observational data to estimate randomized controlled trial generalizability bias. Stat Med. 2013;32:3552-68.

77. Silverman SL. From randomized controlled trials to observational studies. Am J Med. 2009;122:114-20.

78. Yang W, Zilov A, Soewondo P, Bech OM, Sekkal F, Home PD. Observational studies: going beyond the boundaries of randomized controlled trial. Diabetes Res Clin Pract. 2010;88 suppl 1:S3-9.

\section{Submit your next manuscript to BioMed Central and take full advantage of:}

- Convenient online submission

- Thorough peer review

- No space constraints or color figure charges

- Immediate publication on acceptance

- Inclusion in PubMed, CAS, Scopus and Google Scholar

- Research which is freely available for redistribution 